\title{
The motor-semantic meanings of verbs generated by Parkinson's disease patients on/off dopamine medication in a verbal fluency task
}

\author{
Elena Herrera ${ }^{\mathrm{a}, ~}{ }^{\text {, }}$, Beatriz Bermúdez-Margaretto ${ }^{\mathrm{a}}$, Renée Ribacoba ${ }^{\mathrm{b}}$, \\ Fernando Cuetos ${ }^{\text {a }}$ \\ a University of Oviedo, Spain \\ ${ }^{\mathrm{b}}$ Movement Disorders Unit, Central Hospital of Asturias, Spain
}

\section{A R T I C L E I N F O}

\section{Article history:}

Received 27 November 2014

Received in revised form 23 June 2015

Accepted 29 June 2015

Available online 7 July 2015

\section{Keywords:}

Parkinson's disease

Action language

Action fluency

Action processing

Dopamine

\begin{abstract}
A B S T R A C T
The human motor cortex is involved in the planning and execution of movements as well as in the processing of action language, and it is impaired in Parkinson's disease (PD) as a consequence of dopamine degeneration. The aim of this study is to understand the role of dopamine in the processing of action words by looking at PD patients with and without medication. A group of 23 healthy controls and 34 non-demented PD patients participated in an action verbal fluency task on and off dopamine medication. A repeated measures $2 \times 2$ ANOVA was conducted with dopamine (on/off) and type of verb (high and low specificity motor verbs) as independent variables and the number of words generated as a dependent variable. Our results showed that patients on dopamine medication produced a significantly greater number of high specificity motor verbs than low ones. In contrast, patients off dopamine medication generated a similar number of verbs with high and low motor specificity. MANOVA analyses showed a significantly lower production of verbs with high motor specificity on PD patients off medication compared to healthy controls. However, performance of PD patients in the on condition was similar to that found in healthy controls. In conclusion, the dopamine network from basal ganglia to brain motor areas might play a role in retrieving action verbs with specific semantic representations.
\end{abstract}

(C) 2015 Elsevier Ltd. All rights reserved.

\section{Introduction}

The primary function of the human motor cortex is planning and performing complex goal-directed actions, such as manipulating tools and objects. But beyond these executive functions, it is becoming clear that the motor cortex also cooperates with other neural networks to perform cognitive functions (Rizzolatti, Fogassi, \& Gallese, 2002). In fact, numerous studies using a range of behavioral, neuropsychological, electrophysiological and neuroimaging techniques have demonstrated the involvement of sensory-motor systems in language processing in healthy people and brain-damaged patients. In an important study, Hauk, Johnsrude, and Pulvermüller (2004), using functional magnetic resonance imaging (fMRI), found a clear overlap between the frontal regions involved in the processing of action words and the motor areas involved in carrying

\footnotetext{
* Corresponding author. Basic Psychology Laboratory, Department of Psychology, Plaza Feijoo s/n, University of Oviedo, Asturias, Spain.

E-mail address: elenaherreragomez@gmail.com (E. Herrera).
} 
out the real action in healthy volunteers. This theoretical approach named semantic embodiment affirms that language comprehension depends (at least to some extent) on the interaction between the sensory-motor representations and the meanings of the words in the lexical network. In the same way, the production of words with a strong motor component such as action verbs also depends on the activation of specific motor programs stored in cortical areas (Kemmerer \& GonzalezCastillo, 2010; Pulvermüller, Shtyrov, \& Ilmoniemi, 2005). On the other hand, some research using fMRI reported equal activation for action and non-action related stimuli. In the study of Zubicaray, Postle, McMahon, Meredith, and Ashton (2010), they looked to the "mirror" areas located in Broca's area and showed equivalent responses for action words, unrelated words and nonwords, with all of these stimuli showing increased responses relative to visual character strings. Given the controversy, more research is needed to understand the specific role of different brain areas and networks in the processing of normal and impaired language.

The study of patients with motor disturbances helps to understand the relationship between motor and premotor areas of the brain and language processes. Parkinson's disease (PD) is a neurodegenerative disorder not only associated with motor problems but also with some specific language deficits. Numerous investigations have found problems in PD linguistic performance, mainly in the processing of action words. Recent studies have reported deficits in action naming (RodríguezFerreiro, Menéndez, Ribacoba, \& Cuetos, 2009), lexical decision (Angwin, Chenery, Copland, Murdoch, \& Silburn, 2007; Boulenger et al., 2008; Fernandino et al., 2013a), sentence comprehension (Fernandino et al., 2013b; Ibáñez et al., 2013), verb generation (Castner et al., 2008) and verbal fluency (Herrera, Cuetos, \& Ribacoba, 2012; Piatt, Fields, Paolo, Koller, \& Tröster, 1999).

Although there is growing evidence supporting the existence of action language impairment in PD, the mechanisms underpinning this disturbance are still not clear. Some authors argue that the lack of dopamine in the fronto-striatal network affects the motor movements of PD patients and could also be disrupting the processing of action words (Péran et al., 2013). In the study of Péran et al., they used fMRI to investigate cerebral changes after motor improvement due to dopamine treatment in the representation of actions mediated by verb generation and motor imagery. They found that brain activity related to semantics of action is modified by dopaminergic treatment in PD patients. This cerebral reorganization concerns mainly the motor and premotor cortex suggesting an involvement of the putaminal motor loop according to the "motor" theory of verb processing. Interestingly, recent experiments suggest that this disturbance may be related to the semantic meaning of the action words. According to this account, the involvement of motor areas in the processing of verbs increases as the degree of action meaning of words is stronger (Herrera, Cuetos et al., 2012; Herrera, RodríguezFerreiro, \& Cuetos, 2012).

By contrast, some experiments failed to find differences in terms of action meanings in PD patients. For example, Kemmerer, Miller, MacPherson, Huber, and Tranel (2013) evaluated the performance of patients with PD on and off medication on a semantic similarity judgment task using action verbs and non-action verbs. They found similar accuracy for both action and non-action verbs on and off medication, although reaction times were slower than controls. Their data suggest that semantic similarity judgments for both action and non-action verbs are correct but slow in individuals with PD.

Verbal fluency is a classical neuropsychological measure of language production. In particular, phonemic fluency requires individuals to generate lists of words that start with a given letter, while semantic fluency involves generating words to semantic category cues, and action fluency refers to the ability to generate verbs in a limited period of time. Previous findings show that PD patients have impairment in both phonemic and action fluency (Piatt, Fields \& Paolo et al., 1999; Piatt, Fields, Paolo \& Koller et al., 1999; Signorini \& Volpato, 2006), two tasks that mainly involve the frontal and prefrontal areas - areas that are also disrupted as a consequence of the disease. Interestingly, Pereira, Junqué, Bartrés-faz, Martí, and Sala-llonch (2012) found that transcranial direct current stimulation over the dorsolateral prefrontal cortex enhances phonemic fluency in PD (Pereira et al., 2012), suggesting a clear involvement of this area in the searching for words when given a letter cue. Although there are a growing number of studies on verbal fluency decline in PD patients, most of the researchers used phonemic or semantic categories, while action fluency has been much less studied. Given the relationship between motor and premotor areas and action language processing, the action fluency task provides a useful tool to analyze the type of verbs generated by PD patients in terms of word meanings.

The action fluency task yields several measures that are well known to be affected in PD patients, such as a decrease in the number of words generated (Piatt, Fields \& Paolo et al., 1999; Piatt, Fields, Paolo \& Koller et al., 1999; Péran et al., 2013) and an increase in the average lexical frequency of the generated verbs (Herrera, Cuetos et al., 2012). However, to the best of our knowledge, there is no evidence regarding the semantic meaning of those words generated by PD patients in terms of their motor content or the effect of dopamine on the semantics of verbs produced by PD patients.

There is growing evidence for the influence of motor activity in language processing in PD patients (Cardona et al., 2014; Ibáñez et al., 2013), and recent findings support the notion that dopaminergic medication increases striatal-frontal connectivity between basal ganglia and prefrontal cortex (Jahanshahi et al., 2010) and might be affecting the normal processing of action verbs. Thus, the aim of this study is to investigate the effect of dopamine depletion in the retrieval of action verbs attending to the performance of PD patients with and without medication. For this purpose, the semantic meaning of the verbs produced will be analyzed in terms of motor specificity meaning, based on the specificity of the parts of the body needed for doing the action (in contrast to the traditional distinction based on manipulability/graspable actions). Knowing the motor-meanings of those words generated by PD patients could provide information regarding the semantic organization of actions and the involvement of the motor areas in this specific semantic category. We hypothesized that under dopamine deprivation the action words generated by PD patients would have less motor specificity compared to the on dopamine 
condition. Thus, our prediction is that PD patients off dopamine medication will generate a lower number of high specificity motor verbs, while, after dopamine intake, they will produce a greater number of high specificity motor verbs. That means, when motor impairment increases as a consequence of dopamine deprivation, the verbs generated by PD patients present less motor specificity.

\section{Material and methods}

\subsection{Participants}

A group of 34 non-demented PD patients (8 females) with a mean age of 60.60 (range 48-77) and 23 matched healthy controls (13 females) with a mean age of 58.62 (6.32) participated in the study. The PD sample was evaluated twice (on and off dopamine medication). All PD participants were candidates for deep brain stimulation (DBS), and that means that dementia, severe cognitive impairment, depression assessed by Hospital Anxiety and Depression Scale (HADS: Zigmond \& Snaith, 1983) or other psychiatric disorders had been excluded before the experimental evaluation. The inclusion criterion for the surgery was the presence of typical PD following the core assessment program for intracerebral transplantations (CAPIT; Widner, 2010) accompanied by untreatable motor fluctuations and dyskinesia but without signs of atypical PD and at least 30\% motor improvement in the levodopa test. Exclusion criteria included atypical PD syndrome, absence of improvement of motor complication in the on stage, psychiatric disorder, severe depression or cognitive impairment (assessed with a neuropsychological battery). The "off" phase was at least $12 \mathrm{~h}$ without dopamine medication and the "on" stage was around 60 min after intake of the supraliminar dose of dopamine. Not all UPDRS data was recorded for all the sample of PD patients. See Table 1 for demographic details of participants.

\subsection{Materials and procedure}

The neuropsychological battery included the Mini-mental State Examination (MMSE: Folstein, Folstein, \& McHugh, 1975; 20 PD patients and 12 healthy controls), the Montreal Cognitive Assessment (MoCA: Nasreddine et al., 2005 ; 14 PD patients and 11 healthy controls) and an action fluency task. Thus, PD patients performed the MMSE or the MoCA test; all of them achieved scores above the cut-offs for mild cognitive impairment. In the action fluency task, participants were asked to generate as many verbs as they could in $60 \mathrm{~s}$ ("things you can do"). Half of the PD patients were tested first off and then on and half of the participants first on and then off dopamine treatment.

The examiner recorded all the words generated by the participants. Then all verbs (a total of 312 verbs) were categorized into movement, abstract or sensorial verbs by three independent judges. In order to classify the motor content, all movement verbs (a total of 191 different verbs) were included in a 7-scale online questionnaire to have an objective motor-semantic measure of each verb. The questionnaire was completed by 52 normal subjects ( 37 females) with a mean age of 26.45 (range 21-54). In this questionnaire, movement-verbs were categorized on the basis of the nature of the movement, with 7 representing low specificity movements (actions including the whole body) and 1 the high specificity movements (actions produced by small parts of the body such as hands, legs, mouth ...). The instructions for completing the questionnaire were "to score in a range from 1 to 7 the verbs presented if you consider that you need the whole body or specific parts of the body for doing the action". In total, using 3.5 points as the cut-off, 70 verbs were categorized as low specificity motor verbs (3.5-7 in the scale) and 121 as high specificity motor verbs (1-3.5 in the scale). See Table 2 for examples. It is important to clarify that high specificity motor verbs are not always manipulable/graspable actions (for example, write and kiss were categorized as high specificity motor verbs, while write is a manipulable action but kiss is not).

All the verbs produced by each subject were later categorized by the experimenters following the scores obtained in the online questionnaire-scale. The four scores (number of high specificity motor verbs, low specificity motor verbs, abstract and sensory verbs) were obtained for both on and off dopamine medication for each PD patient and for the healthy controls. Before proceeding with the analyses, the normal distribution data were submitted to Kolmogorov-Smirnov tests. Data for movement-verbs were normally distributed $(p>0.05)$, but data relating to abstract and sensory verbs were not adjusted to normality. Therefore, subsequent analyses were performed with the number of motor verbs (high and low motor specificity) produced by the three groups of participants. Given that most of the participants did not produce any sensory or abstract verb in the verbal fluency task and the aimed of the study was to compare the performance of action verbs, sensory and abstract verbs were discarded for the analyses and the interpretation of the data.

Table 1

Demographic characteristics of Parkinson's disease patients (PD) and healthy controls. Mean (SD).

\begin{tabular}{|c|c|c|c|c|c|c|c|c|c|c|}
\hline Group & $\mathrm{N}$ & Age & Years of school & $\begin{array}{l}\text { Years of disease } \\
\text { progression }\end{array}$ & Hours off & Hours on & UPDRS on & UPDRS off & MMSE & MoCA \\
\hline PD & 34 & 60.60 (9.09) & $10.30(4.31)$ & $10.06(3.78)$ & $20.40(6.86)$ & $1.66(-1.17)$ & $21.12(7.75)$ & $55.6(11.87)$ & $28.90(1.04)$ & $25.75(3.74)$ \\
\hline Controls & 23 & $58.62(6.32)$ & $11.48(3.22)$ & - & - & - & - & - & $28.82(0.95)$ & $27.54(1.63)$ \\
\hline
\end{tabular}


Table 2

Examples (translated from Spanish) of verbs with high and low motor specificity generated by PD patients on and off dopamine medication and the specificity-score obtained in the questionnaire in terms of the parts of body implicated in the movement. Scores below 3.5 indicated high specificity motor verbs (very specific part of the body involved in doing the action) and scores above 3.5 indicated low specificity motor verbs (several parts of the body involved in doing the action).

\begin{tabular}{lll}
\hline Verbs with high motor specificity $($ to + ) & Specificity score & Verbs with low motor specificity (to + ) \\
\hline sew & 2 & swim \\
knit & 2 & run \\
bounce & 2 & sleep \\
write & 2 & fight \\
kiss & 2.3 & build \\
eat & 2.4 & climb \\
tie & 2.6 & clean \\
drink & 3 & walk \\
brush & 3 & dance \\
sing & 3.2 & work \\
\hline
\end{tabular}

\section{Results}

Repeated measures $2 \times 2$ ANOVA was run with dopamine (on and off) and motor specificity of verbs (high and low motor specificity) as independent variables and the number of verbs produced by PD patients on each condition as dependent variable. Results indicated a dopamine effect $(F(1,33)=19.58, p<0.001)$ and an interaction between dopamine and motor specificity of verbs $(F(1,33)=10.85, p=0.002)$. No main effect of the motor specificity of verbs was found $(F(1,33)=3.58$, $p=0.067$ ).

Subsequently, one-factor ANOVAs were carried out to compare the number of each type of verb in the two medication conditions. Results showed an effect for the type of verb in the on dopamine condition $(F(1,33)=13.09, p=0.001$, diff. $=2.29)$ but not in the off dopamine condition $(F(1,33)=0.18, p=0.669$, diff. $=0.29)$. Patients on dopamine medication produced a significantly greater number of high specificity motor verbs (7.11) than low specificity motor verbs (4.82). In contrast, patients off dopamine medication generated a similar number of verbs with high (4.55) and low (4.85) motor specificity. Moreover, a dopamine effect was found on the production of verbs with high motor specificity $(F(1,33)=27.78, p<0.001$, diff. $=2.55)$ but not on the production of verbs with low motor specificity $(F(1,33)=0.004, p=0.952$, diff. $=0.029)$, indicating that a greater number of high specificity motor verbs were generated in the on dopamine condition than in the off state, a difference not found for verbs with low motor specificity between both dopamine conditions. See Fig. 1.

Therefore, these results indicated that, when PD patients were under dopamine effects, they generated a greater number of high specificity motor verbs compared to low specificity motor verbs. In contrast, when patients were off dopamine medication, the number of verbs they produced with high and low motor specificity was similar.

A MANOVA was conducted to compare the number of verbs with high and low motor specificity produced by the three groups, PD patients on and off dopamine medication and healthy controls. The analysis resulted in statistical differences between the three groups in their production of high specificity motor verbs $(F(2,91)=7.75, p=0.001)$. Bonferroni post-hoc

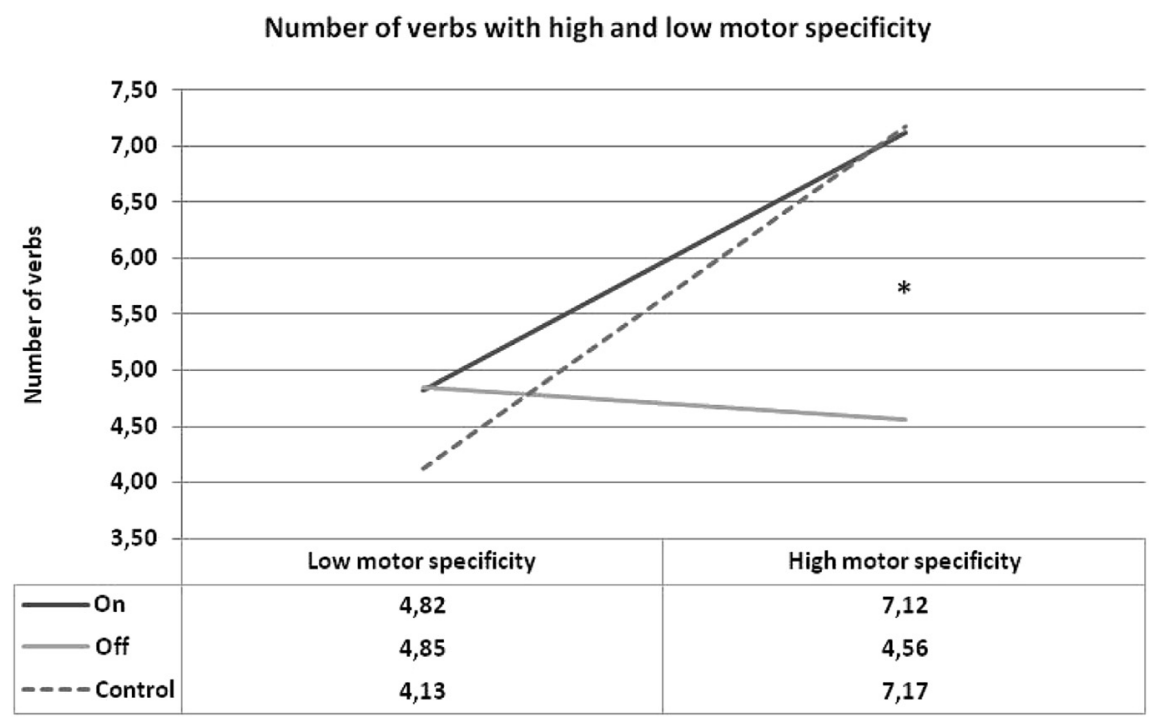

Fig. 1. Number of verbs with high and low motor specificity produced by the three groups, PD patients on dopamine medication (dark line), PD off dopamine medication (light line) and healthy controls (dashed line). Asterisk indicates significant differences in verb production of patients off dopamine medication compared to patients on dopamine medication and healthy controls. 
comparisons determined differences between PD patients on and off dopamine medication $(p=0.002$, diff. $=2.55)$ and between PD patients off dopamine medication and healthy controls $(p=0.006$, diff. $=-2.61)$. Both PD patients under dopamine effects and healthy controls produced a greater number of high specificity motor verbs (7.11 and 7.17, respectively) than PD patients off dopamine medication (4.55). Furthermore, the restoring of dopamine levels resulted in a similar production of high specificity motor verbs among both groups, patients on dopamine medication and healthy controls, without statistical differences between them $(p=1$, diff. $=-0.05)$.

Interestingly, patients off dopamine medication produced a significantly lower number of verbs with high motor specificity compared to patients under dopamine effects and healthy controls. This difficulty in retrieving could be explained by the lack of accessibility of the high specificity motor verbs compared to the low specificity motor verbs in terms of their lexical frequency. New analyses were carried out to discard the effect of this psycholinguistic characteristic of verbs on their production. A MANOVA was conducted to compare the lexical frequency of both types of movement verbs produced by PD patients on and off dopamine medication and healthy controls. No effects of group $(F(2,978)=0.50, p=0.60)$, motor specificity $(F(1,978)=1.49, p=0.22)$ or interaction $(F(2,978)=1.37, p=0.25)$ were found. Analysis for each group separately confirmed that verbs with high and low motor specificity shown similar frequencies in the three groups, patients on medication $(F(1,399)=1.41, p=0.23$, diff. $=4.48)$, off medication $(F(1,319)=0.44, p=0.506$, diff. $=-2.45)$ and healthy controls $(t(1,260)=2.72, p=1$, diff. $=6.16)$. See Fig. 2 .

\section{Discussion}

It has been argued that motor and premotor areas are involved, at least to some extent in language processing (Kiefer \& Pulvermüller, 2012) however, there is also evidence questioning this proposal (Kemmerer et al., 2013). Our aim was to understand the role of dopamine in the processing of action words by comparing the performance of PD patients with and without dopamine medication and healthy controls in an action fluency test, by analyzing the motor semantic content of the verbs generated. To our knowledge, this is the first study investigating the semantic motor-meaning of those verbs generated by PD patients in an action verbal fluency task. Our results showed that, while PD patients off dopamine generated a lower number of high specificity motor verbs in the action fluency task, when dopamine was restored in the on condition, there were a greater number of high specificity motor verbs generated by PD patients. In addition, these results cannot be explained in terms of the low frequency of these verbs, since there is no difference between them in terms of this variable. This suggests a greater involvement of the motor circuitry in those verbs which need more specific semantic representations of movement. Thus, in the off condition, the lack of dopamine under-activates the motor loop disrupting the lexical access to those verbs which require specific semantic representations of movement. Interestingly, in the on condition, the performance of PD patients and healthy controls was similar.

Given that PD patients have a deficit in dopamine networks from basal ganglia to frontal and prefrontal regions and their motor areas are less activated as a consequence of this disturbance, our results might suggest that, when dopamine levels and brain motor areas are functioning well, the verbs generated in an action fluency task are more movement-specific. Numerous experiments support the notion that dopamine increases striatal-frontal connectivity, therefore improving motor movements in PD patients (Jahanshahi et al., 2010), and that the motor system plays a crucial role in action word processing (Hauk et al., 2004; Ibáñez et al., 2013). On the other hand, other experiments also suggest that dopamine reduction in PD patients not only affects language but also a wide range of cortical and subcortical regions, and these changes in dopamine levels may influence other cortical regions during language processing (Copland, McMahon, Silburn, \& de Zubicaray, 2009).

Lexical frequency of verbs with high and low motor specificity

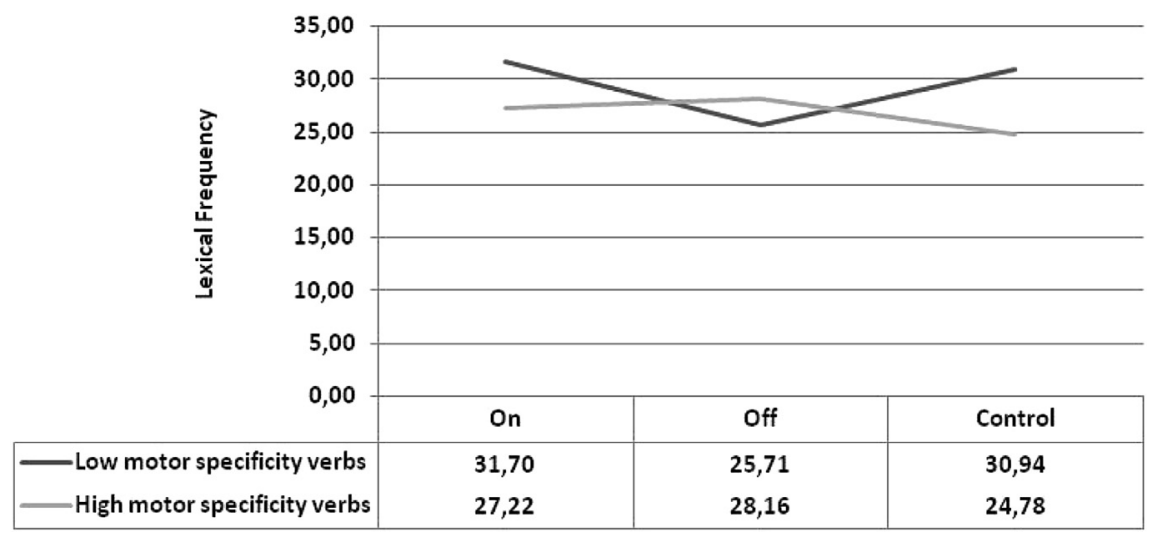

Fig. 2. Lexical frequency values for verbs with low motor specificity (dark line) and verbs with high motor specificity (light line) produced by patients on and off dopamine medication and control group. 
Taken together, our results suggest that the lack of dopamine in the motor system of PD patients is affecting the normal processing of action verbs revealing that those actions with more motor specific movements are more difficult to retrieve for PD patients off dopamine treatment. Some previous findings from action verbal fluency tasks explained the deficit in terms of verb processing impairment being related to decreased frontal lobe function (Piatt, Fields, Paolo, \& Tröster, 1999). In line with this, on the basis of the classical distinction of nouns and verbs in the brain (Damasio \& Tranel, 1993), the deficit of PD patients in the action fluency task was explained by the disruption of the frontal cortex as a consequence of dopamine depletion in the fronto-striatal network, and has been considered a preclinical marker to conversion to dementia (Piatt, Fields \& Paolo et al., 1999; Piatt, Fields, Paolo \& Koller et al., 1999). However, given the new data regarding the relationship between brain motor areas and action language processing in healthy volunteers (for a review see Pulvermüller, 2013) and patients with motor disturbance (Cardona et al., 2014; Herrera, Cuetos et al., 2012; Péran et al., 2013), we thought it would be interesting to analyze the words generated by PD patients in terms of their motor-semantic meaning. Other previous experiments failed to find differences in PD on/off medication using action and non-action verbs. One possible explanation could be that the effect of dopamine in the performance of PD in tasks involving action semantic meanings is only to a mild degree and their comprehension of action meanings is somehow preserved. Another possibility is that this disturbance is not sufficient to prevent patients from achieving a high level of accuracy on the action verbs in the similarity judgment task (Kemmerer et al., 2013).

In a previous experiment by our group, we reported differences in the average lexical frequency of verbs generated by PD patients on and off dopamine medication. Our results showed that PD patients off dopamine treatment generated higher frequency verbs suggesting a deficit in the lexico-semantic access to the less frequent verbs; while in the on dopamine condition the verbs generated were similar to those of controls (Herrera, Cuetos et al., 2012). The results of the present study showed that verbs with high and low motor specificity showed similar frequencies in the three groups. This result confirmed that semantic meaning (and not only frequency of verbs) is the main variable in a PD patient's performance. This result is not contradictory to the previous one by our group, given that for this experiment we only take into account the motor-verbs and the abstract and sensorial were discarded for the analyses. With these new results we argue that more specific analyses should be conducted to understand language deficits in PD population.

These results have important practical implications since they suggest that analyzing action language production in PD patients could be used as an early marker for the evolution of the illness if we assume that the semantics of verbs generated reflect, in some extent, the function of the motor cortex. An alternative explanation could be that high specificity movements are more related with manipulable/graspable tools since viewing graspable tools (but not other objects) activates motorrelated brain regions (Chao \& Martin, 2000; Kalénine et al., 2009). In a recent EEG study, Proverbio, Adorni, and D'Aniello (2011) found the left postcentral gyrus and the left and right premotor cortex were the most significant sources of activity within the first $250 \mathrm{~ms}$ during the observation of manipulable objects. However, these findings are controversial as the relationship between motor knowledge and manipulable objects was not found in a patient with, for example, motor-limb aphasia (Vannuscorps, Andres, \& Pillon, 2014). The importance of our study is that the distinction between high and low specificity motor verbs is based on the specificity of the parts of the body needed for doing the action (in contrast to the traditional distinction based on manipulability/graspable actions). For example, we considered "(to) write" (manipulable action) as a high specificity motor verb but also "(to) sing" (which does not require manipulable action) because both writing and singing need specific parts of the body. The argument proposed here to distinguish between high and low specificity motor verbs is that, as more parts of the body are implicated in the action, the movement becomes less specific and is categorized as low specificity motor verb.

One important limitation of the study is the absence of correlation analyses of motor impairment of PD patients and language production, but in some cases it is difficult to find this data available for our participants. Also, for further experiments, we will consider having neuroimaging data of patients while doing action language tasks to improve the interpretation of the behavioral data.

\section{Conclusions}

In conclusion, our PD patients showed a different pattern of motor-semantic content of the verbs generated during a verbal fluency task depending on whether they were on or off dopamine. Under dopamine effects, patients produced a higher number of verbs referring to high specificity movements compared to the dopamine deprivation condition, in which the same patients produced a significantly fewer number of these verbs. In addition, when dopamine levels were restored, the performance of PD patients was similar to that found in the control group, suggesting an involvement of the motor circuitry in the processing of action words. It seems that for the retrieval of specific action movement verbs, the motor brain areas should be correctly working and, when motor disturbance is stronger as a consequence of dopamine deprivation, PD patients retrieve verbs with less specific motor-semantic representations.

\section{Acknowledgments}

This study was funded by Grant PSI2012-31913, from the Spanish Government. Elena Herrera and Beatriz BermúdezMargaretto were funded by FICYT from the Principado of Asturias Government (BP 09-005 and BP 13028). Special thanks to all the Parkinson's disease patients and their caregivers. 


\section{References}

Angwin, A. J., Chenery, H. J., Copland, D. A., Murdoch, B. E., \& Silburn, P. A. (2007). The speed of lexical activation is altered in Parkinson's disease. Journal of Clinical and Experimental Neuropsychology, 29(1), 73-85. http://dx.doi.org/10.1080/13803390500507188.

Boulenger, V., Mechtouff, L., Thobois, S., Broussolle, E., Jeannerod, M., \& Nazir, T. A. (2008). Word processing in Parkinson's disease is impaired for action verbs but not for concrete nouns. Neuropsychologia, 46(2), 743-756. http://dx.doi.org/10.1016/j.neuropsychologia.2007.10.007.

Cardona, J. F., Kargieman, L., Sinay, V., Gershanik, O., Gelormini, C., Amoruso, L., et al. (2014). How embodied is action language? Neurological evidence from motor diseases. Cognition, 131(2), 311-322. http://dx.doi.org/10.1016/j.cognition.2014.02.001.

Castner, J. E., Chenery, H. J., Silburn, P. A., Coyne, T. J., Sinclair, F., Smith, E. R., et al. (2008). Effects of subthalamic deep brain stimulation on noun/verb generation and selection from competing alternatives in Parkinson's disease. Journal of Neurology, Neurosurgery \& Psychiatry, 79(6), 700-705.

Chao, L. L., \& Martin, A. (2000). Representation of manipulable man-made objects in the dorsal stream. NeuroImage, 12(4), 478-484. http://dx.doi.org/10. 1006/nimg.2000.0635.

Copland, D. A., McMahon, K. L., Silburn, P. A., \& de Zubicaray, G. I. (2009). Dopaminergic neuromodulation of semantic processing: a 4-T fMRI study with levodopa. Cerebral Cortex, 19(11), 2651-2658.

Damasio, A. R., \& Tranel, D. (1993). Nouns and verbs are retrieved with differently distributed neural systems. Proceedings of the National Academy of Sciences of the United States of America, 90(11), 4957-4960. Retrieved from: http://www.ncbi.nlm.nih.gov/pubmed/15037130.

Fernandino, L., Conant, L. L., Binder, J. R., Blindauer, K., Hiner, B., Spangler, K., et al. (2013a). Parkinson's disease disrupts both automatic and controlled processing of action verbs. Brain and Language, 127(1), 65-74. http://dx.doi.org/10.1016/j.bandl.2012.07.008.

Fernandino, L., Conant, L. L., Binder, J. R., Blindauer, K., Hiner, B., Spangler, K., et al. (2013b). Where is the action? Action sentence processing in Parkinson's disease. Neuropsychologia, 51(8), 1510-1517. http://dx.doi.org/10.1016/j.neuropsychologia.2013.04.008.

Folstein, M. F., Folstein, S. E., \& McHugh, P. R. (1975). Mini-mental state. Journal of Psychiatric Research, 12(3), 189-198. http://dx.doi.org/10.1016/00223956(75)90026-6.

Hauk, O., Johnsrude, I., \& Pulvermüller, F. (2004). Somatotopic representation of action words in human motor and premotor cortex. Neuron, 41(2), 301-307. Retrieved from: http://www.ncbi.nlm.nih.gov/pubmed/14741110.

Herrera, E., Cuetos, F., \& Ribacoba, R. (2012). Verbal fluency in Parkinson's disease patients on/off dopamine medication. Neuropsychologia, 50(14), 3636-3640. http://dx.doi.org/10.1016/j.neuropsychologia.2012.09.016.

Herrera, E., Rodríguez-Ferreiro, J., \& Cuetos, F. (2012b). The effect of motion content in action naming by Parkinson's disease patients. Cortex, 48(7), 900-904.

Ibáñez, A., Cardona, J. F., Dos Santos, Y. V., Blenkmann, A., Aravena, P., Roca, M., et al. (2013). Motor-language coupling: direct evidence from early Parkinson's disease and intracranial cortical recordings. Cortex, 49(4), 968-984. http://dx.doi.org/10.1016/j.cortex.2012.02.014.

Jahanshahi, M., Jones, C., Zijlmans, J., Katzenschlager, L., Quinn, N., Frith, C. D., et al. (2010). Dopaminergic modulation of striato-frontal connectivity during motor timing in Parkinson's disease. Brain, 133, 727-745. http://dx.doi.org/10.1093/brain/awq012.

Kalénine, S., Peyrin, C., Pichat, C., Segebarth, C., Bonthoux, F., \& Baciu, M. (2009). Neural specificity of manipulable and non-manipulable object concepts: Influence of object manipulability on conceptual processing in normal adults. NeuroImage, 47, S53. http://dx.doi.org/10.1016/S1053-8119(09)70156-9.

Kemmerer, D., \& Gonzalez-Castillo, J. (2010). The two-level theory of verb meaning: an approach to integrating the semantics of action with the mirror neuron system. Brain and Language, 112(1), 54-76. http://dx.doi.org/10.1016/j.bandl.2008.09.010.

Kemmerer, D., Miller, L., MacPherson, M. K., Huber, J., \& Tranel, D. (2013). An investigation of semantic similarity judgments about action and non-action verbs in Parkinson's disease: implications for the embodied cognition framework. Frontiers in Human Neuroscience, 7.

Kiefer, M., \& Pulvermüller, F. (2012). Conceptual representations in mind and brain: theoretical developments, current evidence and future directions. Cortex a Journal Devoted to the Study of the Nervous System and Behavior, 48(7), 805-825. http://dx.doi.org/10.1016/j.cortex.2011.04.006.

Nasreddine, Z. S., Phillips, N. A., Bédirian, V., Charbonneau, S., Whitehead, V., Collin, I., et al. (2005). The Montreal Cognitive Assessment, MoCA: a brief screening tool for mild cognitive impairment. Journal of the American Geriatrics Society, 53(4), 695-699.

Péran, P., Nemmi, F., Méligne, D., Cardebat, D., Peppe, A., Rascol, O., et al. (2013). Effect of levodopa on both verbal and motor representations of action in Parkinson's disease: a fMRI study. Brain and Language, 125(3), 324-329. http://dx.doi.org/10.1016/j.bandl.2012.06.001.

Pereira, J. B., Junqué, C., Bartrés-faz, D., Martí, M. J., \& Sala-llonch, R. (2012). Brain stimulation modulation of verbal fluency networks by transcranial direct current stimulation (tDCS) in Parkinson's disease. Brain Stimulation, 1-8. http://dx.doi.org/10.1016/j.brs.2012.01.006.

Piatt, A. L., Fields, J. A., Paolo, A. M., \& Tröster, A. I. (1999). Action (verb naming) fluency as an executive function measure: convergent and divergent evidence of validity. Neuropsychologia, 37(13), 1499-1503. Retrieved from: http://www.ncbi.nlm.nih.gov/pubmed/10617270.

Piatt, A. L., Fields, J. A., Paolo, A. M., Koller, W. C., \& Tröster, A. I. (1999). Lexical, semantic, and action verbal fluency in Parkinson's disease with and without dementia. Journal of Clinical and Experimental Neuropsychology, 21(4), 435-443. http://dx.doi.org/10.5555/jcen.21.4.435.885.

Proverbio, A. M., Adorni, R., \& D'Aniello, G. E. (2011). $250 \mathrm{~ms}$ to code for action affordance during observation of manipulable objects. Neuropsychologia, 49(9), 2711-2717. http://dx.doi.org/10.1016/j.neuropsychologia.2011.05.019.

Pulvermüller, F. (2013). Semantic embodiment, disembodiment or misembodiment? In search of meaning in modules and neuron circuits. Brain and Language, 127(1), 86-103. http://dx.doi.org/10.1016/j.bandl.2013.05.015.

Pulvermüller, F., Shtyrov, Y., \& Ilmoniemi, R. (2005). Brain signatures of meaning access in action word recognition. Journal of Cognitive Neuroscience, 17(6), 884-892. http://dx.doi.org/10.1162/0898929054021111.

Rizzolatti, G., Fogassi, L., \& Gallese, V. (2002). Motor and cognitive functions of the ventral premotor cortex. Current Opinion in Neurobiology, 12(2), 149-154. http://dx.doi.org/10.1016/S0959-4388(02)00308-2.

Rodríguez-Ferreiro, J., Menéndez, M., Ribacoba, R., \& Cuetos, F. (2009). Action naming is impaired in Parkinson disease patients. Neuropsychologia, 47(14), 3271-3274. http://dx.doi.org/10.1016/j.neuropsychologia.2009.07.007.

Signorini, M., \& Volpato, C. (2006). Action fluency in Parkinson's disease: a follow-up study. Movement Disorders, 21(4), 467-472.

Vannuscorps, G., Andres, M., \& Pillon, A. (2014). Is motor knowledge part and parcel of the concepts of manipulable artifacts? Clues from a case of upper limb aplasia. Brain and Cognition, 84(1), 132-140. http://dx.doi.org/10.1016/j.bandc.2013.11.009.

Widner, H. (2010). Encyclopedia of movement disorders (pp. 182-187). Elsevier. http://dx.doi.org/10.1016/B978-0-12-374105-9.00445-7.

Zigmond, A. S., \& Snaith, R. P. (1983). The hospital anxiety and depression scale. Acta Psychiatrica Scandinavica, 67(6), 361-370. http://dx.doi.org/10.1111/j. 1600-0447.1983.tb09716.x.

Zubicaray, G., Postle, N., McMahon, K., Meredith, M., \& Ashton, R. (2010). Mirror neurons, the representation of word meaning, and the foot of the third left frontal convolution. Brain and language, 112(1), 77-84. 